\title{
Magnetic field effects on spatial relaxation of swarm particles in the idealized steady-state Townsend experiment
}

\author{
B. Li, ${ }^{1}$ R. E. Robson, ${ }^{2}$ and R. D. White ${ }^{2}$ \\ ${ }^{1}$ School of Physics, University of Sydney, NSW 2006, Australia \\ ${ }^{2}$ School of Mathematical and Physical Sciences, James Cook University, QLD 4810, Australia
}

(Received 4 April 2006; published 29 August 2006)

\begin{abstract}
The effect of a magnetic field at right angles to an electric field on spatial relaxation of a swarm of charged particles emitted by a plane source into a gas — the idealized steady-state Townsend experiment—is examined. The Boltzmann equation is solved using an adaptation of the "two-temperature" moment method, involving a Burnett function representation of the velocity distribution function, a technique which is valid for charged particles of arbitrary mass and is intrinsically of a "multiterm" nature. Results are presented for electrons in model and real gases, and are benchmarked against an exact analytical solution of the Boltzmann equation for a particular collision model. The application of a magnetic field significantly alters the relaxation profiles: in general, it can both enhance or retard spatial relaxation of transport properties. For methane gas, a multiterm analysis is essential to correctly account for the relaxation near the source, even though a two-term approximation may be sufficient when the magnetic field is sufficiently strong and hydrodynamic conditions dominate.
\end{abstract}

DOI: 10.1103/PhysRevE.74.026405

PACS number(s): 52.25.Fi, 52.25.Dg, 51.10.+y, 51.60.+a

\section{INTRODUCTION}

In the last decade, significant advances have been made in the kinetic modeling of charged-particle swarms emitted from a plane source into an infinite gas-the idealized steady-state Townsend (SST) experiment-where the main interest is in the way the applied electric field controls the qualitative and quantitative characteristics of the spatial relaxation [1]. ${ }^{1}$ Most work has focused on electrons, through various "multiterm" methods of solution of the Boltzmann equation [2-7], involving representation of the distribution function in directions $(\theta, \varphi)$ in velocity space in terms of spherical harmonics $Y_{m}^{(l)}(\theta, \varphi)$ beyond $l=1$ (the "two-term" approximation). ${ }^{2}$ In those numerical codes specific to electrons, this angular decomposition is generally followed by discretization in speed [2-5]. However, in the "twotemperature" moment method [8], as adapted to the SST problem by Robson and co-workers [6,7], the speed dependence is accounted for by an expansion in Sonine polynomials with a Maxwellian weight function at some basis temperature different from the gas temperature, leading to an overall Burnett function decomposition of the velocity distribution function. This method, originally developed by Viehland and Mason [9], is most efficient for the calculation of

\footnotetext{
${ }^{1}$ A "swarm" is the test particle or free diffusion limit of a plasma, where charged particle densities are so low that the Debye length is larger than the relevant macroscopic length and none of the collective effects characteristic of a plasma (Debye length much smaller than the relevant macroscopic dimension) are in evidence. In particular, transport processes are collisionally dominated and any space charge fields are negligible in comparison with the external fields. Many of the papers cited in the present paper claim to deal with plasmas, but like the present work, are in fact confined to the swarm limit.

${ }^{2}$ In the usual SST experiment, the electric field normal to the plane source provides an axis of symmetry, and only spherical harmonics with $m=0$-i.e., Legendre polynomials $P_{l}(\theta)$-are required.
}

velocity averages, or "moments" of the distribution function, and can be used for particles of arbitrary mass-i.e., both ions and electrons. Furthermore, it is automatically multiterm in the sense described above. Once the velocity dependence has been accounted for, through either of these two ways ${ }^{3}$ the remaining space dependence can be dealt with either by discretization at a finite number of points or through an eigenfunction expansion method. The former procedure, while somewhat cumbersome, furnishes a comprehensive picture of the spatial dependence in all regions, while the latter, though in principle capable of providing the same information, offers the most efficient means of analyzing the asymptotic spatial relaxation regime, far downstream from the source $[6,7]$. In this paper we generalize our earlier analysis with the two-temperature moment method to solve the Boltzmann equation in the presence of orthogonal electric and magnetic fields, using both spatial finite-difference and eigenfunction techniques.

Charged-particle transport in gases under the influence of crossed electric and magnetic fields has long been of interest, with many scientific and technological applications. To name a few: low-temperature magnetron plasma discharges which are widely used in plasma processing industry [10], the design of multiwire drift chambers for detection particles in high-energy accelerators [11], meteor trails in the Earth's atmosphere [12], gas lasers [13], and swarm experiments in which a magnetic field is introduced with the aim of refining the accuracy of low-energy scattering cross-section data [14].

These applications and experiments are often modeled assuming hydrodynamic conditions, in which the spatial dependence of the charged particle distribution function and its velocity moments, such as the particle flux, are projected entirely onto the number density, typically through a density-

\footnotetext{
${ }^{3}$ Polynomial and "discrete ordinate" representations have long been known to be mathematically equivalent—see, e.g., [53]— although in practice in numerical calculations, there may be advantages in using one method over the other.
} 
gradient expansion: Fick's law of diffusion is an example of a flux-gradient relation valid only in the hydrodynamic regime. The hydrodynamic assumption may be invalid for a number of reasons, such as the presence of sources and sinks $[15,16]$, even for some simple situations, such as the intrinsically nonhydrodynamic idealized SST experiment dealt with in this work. Fick's law is then both quantitatively and qualitatively incorrect, and transport coefficients have no role to play [17]. In these circumstances, an understanding of the mechanisms governing spatial relaxation is best afforded by a full solution of the Boltzmann equation (1), as detailed in this work. This issue has been addressed by many authors in the context of the electrical-field-only case-e.g., Refs. [18-20].

Crossed electric and magnetic fields introduce some complexity into the tensor analysis - there is no single axis of symmetry and a simple Legendre polynomial expansion is no longer valid. Perhaps for this reason, the two-term approximation has hitherto dominated plasma discharge modeling [21-23]. Very recently, however, a multiterm approach for cylindrical magnetron discharges in crossed electric and magnetic fields has been developed [24], extending the previously developed nonhydrodynamic multiterm analysis for an electric field only [25] and the hydrodynamic transport theory when both fields are present [26]. The approach was then applied to model the electron component of an argon magnetron discharge $[27,28]$, and undoubtedly this work provides the most sophisticated such analysis to date. However, a comprehensive multiterm theory emphasizing the fundamental physics of charged-particle spatial relaxation in a crossed-field configuration is still lacking, and this is addressed in the present work.

In the case of an electric field only, previous work on the idealized SST experiment $[2,3,6,7]$ showed that for electrons undergoing particle-conserving (elastic and inelastic) collisions, the spatial relaxation characteristics are strongly controlled by competition between elastic and inelastic collisional energy losses, which play markedly different roles. On the one hand, elastic collisions always produce monotonic relaxation to a spatially uniform state, by virtue of continuous energy losses in such processes. On the other hand, a charged particle suffers a discrete loss of the inelastic threshold energy when it undergoes an inelastic collision. In the absence of elastic collisions, the charged particle accumulates energy from the electric field until it experiences a sudden loss of energy, upon reaching the threshold energy. This process of energy transfer would be repeated without limit if elastic collisions could be "switched off," and the transport properties would thus evolve periodically in space. However, elastic collisions are always present and tend to damp this oscillatory behavior and broaden the peaks [6]. Therefore, spatial relaxation is characterized by monotonic decay or weakly damped periodic decay if elastic or inelastic collisions dominate energy losses, respectively, or heavily damped oscillatory decay when losses of energy via both kinds of collisions are important. The relaxation is thus characterized by a relaxation length and a relaxation period if oscillations exist. The situation is analogous to a damped pendulum.

The presence of a magnetic field orthogonal to the electric field is known to produce significant changes in the relax- ation profile for electrons in neon-e.g., shortening the relaxation length and enlarging the relaxation period for low electric field strengths, as studied in the context of a twoterm analysis [21]. In this work, we extend our earlier multiterm approaches $[6,7]$ to include a magnetic field at right angles with the electric field and develop a formalism suitable for dealing with both ions and electrons. We present relaxation results for electrons undergoing conservative collisions, using both a model and a real gas, here methane [14], as an example. The aim of using model gases is to isolate the explicit magnetic field effects from effects introduced by various collision processes present in real gases, thus enabling us to illustrate the fundamental effects of the magnetic field on spatial relaxation characteristics. We use methane because of the special properties of its cross sections requiring a distinctly "multiterm" analysis [7,26,29-31]. Both cases have been extensively discussed for hydrodynamic [32-34] and nonhydrodynamic conditions [7], respectively, though the latter was for an electric field only.

The structure of this paper is as follows. In Sec. II the kinetic theory of spatially inhomogeneous swarms is summarized, focusing on the Burnett function representation of the Boltzmann equation, and boundary conditions, as well as the eigenfunction decomposition in space. Numerical calculations are performed and the effects of magnetic field on spatial relaxation characteristics of transport properties are demonstrated and discussed in Sec. III. Our results are summarized in Sec. IV.

\section{KINETIC THEORY}

The kinetic theory of inhomogeneous swarms in gases has been discussed extensively in the literature $[1,6,7,35]$. In this section we discuss the solution of the Boltzmann equation using a two-temperature moment technique and the associated boundary conditions. To complement the numerical studies and gain more physical insight, we further present an eigenvalue analysis of the Boltzmann equation, with focus on eigenvalue spectra.

\section{A. Governing equation}

Consider an idealized SST experiment with plane-parallel geometry, as shown schematically in Fig. 1. A plane source emits charged particles with charge $q$ at a steady rate into an infinite gas between anode and cathode, under the influence of crossed static electric and magnetic fields $\boldsymbol{E}$ and $\boldsymbol{B}$, which are chosen in this work such that $q \boldsymbol{E}$ and $q \boldsymbol{B}$ are in the $z$ and $y$ directions, respectively. There exists a steady state in which properties are independent of time and vary with position only in the $z$ direction. The density of the charged particles is so low that they can be treated in the swarm limit. The evolution of the swarm charged particles is governed by the Boltzmann equation

$$
\left[J+\frac{q}{m}\left((\boldsymbol{E}+\boldsymbol{c} \times \boldsymbol{B}) \cdot \frac{\partial}{\partial \boldsymbol{c}}+c_{z} \frac{\partial}{\partial z}\right] f(z, \boldsymbol{c})=0,\right.
$$

where $m$ is mass of the swarm particle, $f(z, c)$ is the particle distribution function at coordinate $z$ and velocity $c, J$ is the 


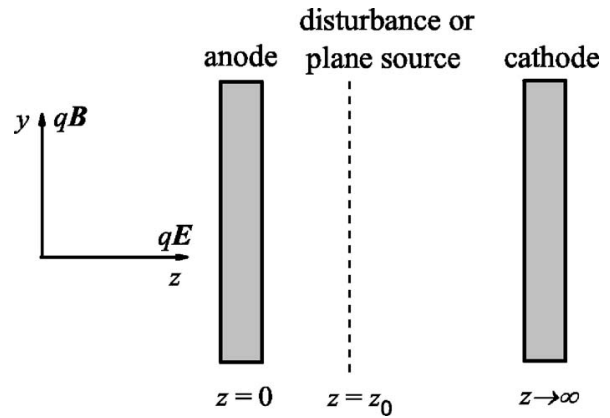

FIG. 1. Schematic representation of the idealized state-state Townsend experiment. Charged particles emitted at a constant rate from an infinite plane source at $z=z_{0}$ interact with the background neutral gas under static external electric and magnetic fields and evolve downstream $z \geqslant z_{0}$.

linear collision operator describing charged-particle-gasmolecule interactions. We consider conservative collision processes only, and the Wang-Chang-Uhlenbeck-de Boer semiclassical collision operator $[15,36,37]$ is used for $J$.

\section{B. Velocity angular dependence: Spherical harmonics decomposition}

In the presence of a magnetic field, decomposition of the angular dependence in velocity space through spherical harmonics not a Legendre polynomial is required, irrespective of the nature of swarm-particle transport being hydrodynamic [26] or nonhydrodynamic [24]. The first step in our solution of Eq. (1) is spherical harmonic decomposition of $f(z, c)$ :

$$
f(z, c)=\sum_{l=0}^{\infty} \sum_{m=-l}^{l} f_{m}^{(l)}(z, c) Y_{m}^{[l]}(\hat{\boldsymbol{c}}),
$$

where $Y_{m}^{[l]}$ are spherical harmonics and $\hat{\boldsymbol{c}}$ is the angle between $\boldsymbol{c}$ and $\boldsymbol{E}$. For practical purposes, truncation of the infinite series in Eq. (2) at some finite value $l=l_{\max }$ is needed. Here $l_{\max }$ is a free parameter set by the convergence requirement, so our formalism is of "multiterm" nature.

Substitution of the expansion (2) into (1), premultiplying by $Y_{m}^{(l)}$, and integrating over all angles yields the following hierarchy of coupled integro-differential equations:

$$
\begin{aligned}
& \sum_{l^{\prime}=0}^{\infty} \sum_{m^{\prime}=-l^{\prime}}^{l^{\prime}}\left(J^{l} \delta_{l^{\prime} l} \delta_{m^{\prime} m}-i a\left(l^{\prime} m 10 \mid \operatorname{lm}\right)\left\langle l\left\|\partial_{c}^{[1]}\right\| l^{\prime}\right\rangle\right. \\
& +\frac{\Omega}{2}\left[\sqrt{(l-m)(l+m+1)} \delta_{m^{\prime} m+1}\right. \\
& \left.-\sqrt{(l+m)(l-m+1)} \delta_{m^{\prime} m-1}\right] \delta_{l^{\prime} l}-i\left(l^{\prime} m 10 \mid l m\right) \\
& \left.\times\left\langle l\left\|c^{[1]}\right\| l^{\prime}\right\rangle \delta_{m^{\prime} m} \frac{\partial}{\partial z}\right) f_{m^{\prime}}^{(l)}(z, c)=0,
\end{aligned}
$$

where $a=q E / m$ and $\Omega=q B / m$ are charged-particle acceleration and gyro frequency, respectively. The reduced matrix elements $J^{l},\left\langle l\left\|\partial_{c}^{[1]}\right\| l^{\prime}\right\rangle$, and $\left\langle l\left\|c^{[1]}\right\| l^{\prime}\right\rangle$ are given by Eqs. (18),
(23), and (24), respectively of Ref. [31]. The quantity $\left(l^{\prime} m 10 \mid l m\right)$ denotes a Clebsch-Gordan coefficient.

Transport properties-for instance, average velocity and mean energy in terms of $f_{m}^{(l)}$-are given by, respectively,

$$
\begin{aligned}
& v_{x}(z)=\sqrt{\frac{4 \pi}{3}} \frac{1}{n(z)} \int_{0}^{\infty} c f_{1}^{(1)}(z, c) c^{2} d c, \\
& v_{z}(z)=\sqrt{\frac{4 \pi}{3}} \frac{1}{n(z)} \int_{0}^{\infty} c f_{0}^{(1)}(z, c) c^{2} d c, \\
& \varepsilon(z)=\sqrt{4 \pi} \frac{1}{n(z)} \int_{0}^{\infty} \frac{1}{2} m c^{2} f_{0}^{(0)}(z, c) c^{2} d c,
\end{aligned}
$$

where $n(z)$ is the charge-particle number density given by

$$
n(z)=\sqrt{4 \pi} \int_{0}^{\infty} f_{0}^{(0)}(z, c) c^{2} d c .
$$

\section{Speed dependence: Sonine polynomial representation}

The speed dependence of $f_{m}^{(l)}$ is further expanded in terms of Sonine polynomials:

$$
f_{m}^{(l)}(z, c)=w(\alpha, c) \sum_{\nu=0}^{\infty} F(\nu l m ; \alpha, z) R_{\nu l}(\alpha c),
$$

where

$$
\begin{gathered}
R_{\nu l}(\alpha c)=N_{\nu l}\left(\frac{\alpha c}{\sqrt{2}}\right)^{l} S_{l+1 / 2}^{(\nu)}\left(\frac{\alpha^{2} c^{2}}{2}\right), \\
N_{\nu l}^{2}=\frac{2 \pi^{3 / 2} \nu !}{\Gamma(\nu+l+3 / 2)},
\end{gathered}
$$

and $S_{l+1 / 2}^{(\nu)}$ are Sonine polynomials. The weight function $w(\alpha, c)$ is a Maxwellian

$$
\begin{gathered}
w(\alpha, c)=\left(\frac{\alpha^{2}}{2 \pi}\right)^{3 / 2} \exp \left[-\frac{\alpha^{2} c^{2}}{2}\right], \\
\alpha^{2}=\frac{m}{k T_{b}}
\end{gathered}
$$

where $T_{b}$ is an arbitrary basis temperature which is different from the gas temperature $T_{0}$. The combination of Sonine polynomials and spherical harmonics leads to Burnett functions [38]. In the parlance of modern kinetic theory [8,29], we thus have a "two-temperature" Burnett function representation of the Boltzmann equation. As with previous work in the hydrodynamic regime $[15,31,39]$ and nonhydrodynamic regime for electric field only $[6,7]$, the Burnett function expansion method seems best suited for a unified treatment of both ions and electrons in the nonhydrodynamic regime when both electric and magnetic fields are present. Expressions for matrix elements of $J$ in this basis set and associated computer codes developed in connection with nonhydrody- 
namic transport calculations in our previous work [7] can be directly adapted to the present problem.

Substituting Eq. (8) into Eq. (3) and multiplying by $R_{\nu l}(\alpha c) c^{2}$, integrating over all speeds, and using the orthogonality of the $R_{\nu l}$ functions with respect to the Maxwellian weight function yields the following system of coupled differential equations for the moments $F\left(\nu^{\prime} l^{\prime} m^{\prime} ; \alpha, z\right)$ :

$$
\begin{gathered}
\sum_{\nu^{\prime}=0}^{\infty} \sum_{l^{\prime}=0}^{\infty} \sum_{m^{\prime}=-l^{\prime}}^{l^{\prime}}\left[n_{0} J_{\nu \nu^{\prime}}^{l}(\alpha) \delta_{l^{\prime} l} \delta_{m^{\prime} m}+i a \alpha\left(l^{\prime} m 10 \mid l m\right)\right. \\
\times\left\langle\nu l\left\|K^{[1]}\right\| \nu^{\prime} l^{\prime}\right\rangle \delta_{m^{\prime} m}+\frac{\Omega}{2}\left[\sqrt{(l-m)(l+m+1)} \delta_{m^{\prime} m+1}\right. \\
\left.-\sqrt{(l+m)(l-m+1)} \delta_{m^{\prime} m-1}\right] \delta_{l^{\prime} l} \delta_{\nu^{\prime} \nu}-i \frac{1}{\alpha}\left(l^{\prime} m 10 \mid l m\right) \\
\left.\times\left\langle\nu l\left\|\alpha c^{[1]}\right\| \nu^{\prime} l^{\prime}\right\rangle \delta_{m^{\prime} m} \frac{\partial}{\partial z}\right] F\left(\nu^{\prime} l^{\prime} m^{\prime} ; \alpha, z\right)=0, \\
(\nu, l)=0,1,2, \ldots, \infty, \\
m=-l,-l+1, \ldots, l,
\end{gathered}
$$

where $n_{0}$ is the neutral gas number density. The reduced matrix elements $J_{\nu \nu^{\prime}}^{l}(\alpha),\left\langle\nu l\left\|\alpha c^{[1]}\right\| \nu^{\prime} l^{\prime}\right\rangle$, and $\left\langle\nu l\left\|K^{[1]}\right\| \nu^{\prime} l^{\prime}\right\rangle$ of the collision operator, velocity, and velocity derivative are given by Eqs. (11), (12a), and (12b) of Ref. [37], respectively.

The quantities of interest in terms of the calculated moments are

$$
\begin{gathered}
v_{x}(z)=\frac{\sqrt{2}}{\alpha} \frac{\operatorname{Im}[F(011 ; \alpha, z)]}{F(000 ; \alpha ; z)}, \\
v_{z}(z)=-\frac{1}{\alpha} \frac{\operatorname{Im}[F(010 ; \alpha, z)]}{F(000 ; \alpha ; z)}, \\
\varepsilon(z)=\frac{3}{2} k T_{b}\left[1-\sqrt{\frac{2}{3}} \frac{F(100 ; \alpha, z)}{F(000 ; \alpha, z)}\right], \\
n(z)=F(000 ; \alpha, z) .
\end{gathered}
$$

As in our early work [7], $T_{b}$ is assumed constant in space.

\section{Boundary conditions}

We apply similar boundary conditions as in our previous work [7]. At the $z=z_{0}$ plane, a disturbing source is applied to the charged-particle distribution functions via a drifted Maxwellian velocity distribution:

$$
f_{D M}(\boldsymbol{c})=A\left(\frac{m}{2 \pi k T_{i}}\right)^{3 / 2} \exp \left[-\frac{m\left(\boldsymbol{c}-\boldsymbol{v}_{i}\right)^{2}}{2 k T_{i}}\right],
$$

with prescribed temperature and drift velocity parameters $T_{i}$ and $v_{i}$, respectively, and amplitude $A$. The boundary conditions at the $z=z_{0}$ plane are on the odd spherical harmonic projections [7]:

$$
f_{m}^{(l)}\left(z_{0}, c\right)=f_{D M, m}^{(l)}(c) \quad[l=1,3, \ldots, \text { but }(l \neq 1, m \neq 0)] .
$$

Due to conservation of charged-particle flux $\Gamma_{z}=n v_{z}$ in the $z$ direction, upon applying relation (5) we have, in addition,

$$
f_{m}^{(l)}\left(z_{0}, c\right)=f_{s, m}^{(l)}(c) \quad(l=1, m=0),
$$

where $f_{s, m}^{(l)}(c)$ is the distribution under steady-state hydrodynamic conditions. It is through the relation (20) that the parameter $A$ is fixed.

In the asymptotic region far downstream from $z=z_{0}$, the distribution function becomes spatially independent since we consider only conservative collisional processes-i.e.,

$$
\frac{\partial}{\partial z} f_{m}^{(l)}(z \rightarrow \infty, c)=0
$$

As is usual with these problems, the infinite half-space is approximated by a finite mesh on a region $\left[z_{0}, z_{\max }\right]$ and the upper limit $z_{\max }$ is increased until solutions do not vary outside the prescribed accuracy limits. The "boundary condition" (21) at infinity is then written in terms of the even spherical harmonic components as [7]

$$
\frac{\partial}{\partial z} f_{m}^{(l)}\left(z_{\max }, c\right)=0 \quad(l=0,2, \ldots) .
$$

It must be emphasized that this requirement does not represent the condition at any physical boundary.

In terms of Burnett function expansions, the boundary conditions for the spherical harmonic projections (19), (20), and (22) now become

$$
\begin{gathered}
F\left(\nu l m ; \alpha, z_{0}\right)=F_{D M}(\nu l m) \\
{[l=1,3, \ldots, \text { but }(l \neq 1, m \neq 0, \nu \neq 0)],} \\
F\left(\nu l m ; \alpha, z_{0}\right)=F_{s}(\nu l m) \quad(l=1, m=0, \nu=0), \\
\frac{\partial}{\partial z} F\left(\nu l m ; \alpha, z_{\max }\right)=0 \quad(l=0,2, \ldots),
\end{gathered}
$$

where $F_{s}$ and $F_{D M}$ denote the moments corresponding to $f_{s, m}^{(l)}$ and $f_{D M, m}^{(l)}$, respectively.

\section{E. Numerical techniques}

We employ similar techniques as for the electric field only case [7] to solve Eqs. (13) and (23)-(25). However, due to the introduction of the additional $m$ index required by the presence of the magnetic field, the matrix size is larger by a factor of $\left(l_{\max }+1\right)$ than when only the electric field is present. For the results represented below, an accuracy of 1\%-2\% has been achieved.

\section{F. Eigenvalue spectra analysis}

In our previous work [6], we developed an eigenvalue theory of the spatially inhomogeneous Boltzmann equation. This theory was then applied to study spatial relaxation of a 
nonhydrodynamic electron swarm in idealized SST experiments, under a spatially uniform electrostatic field. In the present work, we generalize the previous eigenvalue theory to include both electric and magnetic fields which are constant and orthogonal to each other.

The eigenvalue problem is [6]

$$
(M+K) \psi=0
$$

where

$$
M=J+a \frac{\partial}{\partial c_{z}}+\Omega\left(-c_{z} \frac{\partial}{\partial c_{x}}+c_{x} \frac{\partial}{\partial c_{z}}\right)
$$

$K$ is the eigenvalue, and $\psi$ the corresponding eigenfunction. The eigenvalue spectra are generally discrete and so can be represented as $\left\{K_{n}, n=0,1,2, \ldots\right\}$. Moreover, the spectra are complex usually and three types of eigenmodes exist: $K_{0}$ $=0, \operatorname{Re}\left(K_{n}\right)>0$, and $\operatorname{Re}\left(K_{n}\right)<0$. The existence of the different types of eigenmodes indicates mathematically that the problem is of a boundary value nature, as addressed in Sec. II D. Because here we are interested in spatial relaxation in the downstream region, only the $K_{0}=0$ and $\operatorname{Re}\left(K_{n}\right)<0$ eigenmodes are then taken into account. We further assume the eigenvalue spectra $\left\{K_{n}, n=0,1,2, \ldots\right\}$ are arranged in a way such that $\left|\operatorname{Re}\left(K_{n}\right)\right|$ increases with $n$. is [6]

For particle-conserving collisions, the lowest eigenvalue

$$
K_{0}=0,
$$

and $\psi_{0}$ is essentially the steady-state distribution function satisfying

$$
M \psi_{0}=0
$$

The distribution $f(z, c)$ governed by Eq. (1) approaches asymptotically the state represented by $\psi_{0}$, and this occurs at a rate controlled by $K_{1}$, the eigenvalue with the lowest (in magnitude) nonzero real part. This eigenvalue is thus of particular interest.

For the special case of elastic collision interactions only, $K_{n}(n \geqslant 1)$ is real and negative. In the presence of inelastic collisions, under certain conditions $K_{1}$ together with some other $K_{n}$ is complex. The complex feature of $K_{1}$ indicates that the asymptotic relaxation of $f(z, c)$ and its moments are oscillatory with a period $P=2 \pi / \operatorname{Im}\left(K_{1}\right)$ and the approach to the spatially uniform state occurs in a few multiples of the characteristic relaxation length $\Lambda$ given by $\Lambda=1 /\left|\operatorname{Re}\left(K_{1}\right)\right|$.

Equation (26) associated with the $M$ operator given by Eq. (27) is represented in terms of Burnett functions. The numerical solution for $K_{n}$ is carried out using a method similar to that described in Ref. [6].

For the Maxwell elastic collision model and charged particles in a cold gas, analytical solutions of the eigenvalues from Eq. (26) are available (see the Appendix). The eigenvalue spectra are given by

$$
K_{n}=-\frac{6 n(2 n+3)}{(4 n+3)^{2}} \frac{m}{m_{0}} \frac{\nu_{m}^{2}}{a}\left[1+\left(\frac{\Omega}{\nu_{m}}\right)^{2}\right] \quad(n=0,1,2, \ldots)
$$

where $m_{0}$ is the mass of the neutral molecule and $\nu_{m}$ the momentum transfer collision frequency which is related to momentum transfer cross section $\sigma_{m}$ via $\nu_{m}=n_{0} c \sigma_{m}$. The relation (30) shows clearly that the eigenvalues are discrete. Relation (30) implies that when a magnetic field is present, the eigenvalues are enlarged by a factor of $\left[1+\left(\Omega / \nu_{m}\right)^{2}\right]$; consequently, the characteristic relaxation length $\Lambda$ is reduced by the same factor. Spatial relaxation of transport properties thus proceeds faster than the magnetic-field-free case.

One may be tempted to extend the equivalent electric field concept as used in the spatially homogeneous case [40-44] under a two-term approximation to describe the effects of the applied orthogonal magnetic field under nonhydrodynamic conditions. The presence of spatial derivatives in Eq. (1), however, prevents such a representation.

\section{APPLICATIONS}

In this section, we study electron transport in model and real gases. (The procedure could just as easily be applied to ions-no changes are required in the theory and associated computer codes.) Both eigenvalue analysis and numerical calculations are performed using Eqs. (26), (13), and (23)(25). In what follows, the reduced electric and magnetic field strengths $E / n_{0}$ and $B / n_{0}$ are in units $\mathrm{Td}$ and $\mathrm{Hx}$, respectively, where $1 \mathrm{Td}=10^{-21} \mathrm{~V} \mathrm{~m}^{2}$ and $1 \mathrm{Hx}=10^{-27} \mathrm{~T} \mathrm{~m}^{3}$. It is worth noting at the outset that the values of $B / n_{0}$ may be as much as several $\mathrm{kHx}$ or larger in actual plasma processing devices, highlighting the need for nonhydrodynamic studies $[24,45]$, such as the following. The spatial position and the eigenvalues are normalized to a representative mean free path

$$
\lambda=\frac{1}{\sqrt{2} n_{0} \sigma_{0}},
$$

via $K^{\star}=\lambda K$ and $z^{\star}=z / \lambda$, respectively, where $\sigma_{0}=1 \AA^{2}$ is a representative cross section. For simplicity, we set $z_{0}=0$ from now on.

\section{A. Maxwell model}

As discussed in Sec. II F, for Maxwell model electrons in a cold gas, analytical eigenvalue spectra are given by Eq. (30). To validate the theory and computer codes, comparison of the eigenvalues is made between analytical and numerical results. The Maxwell model under consideration is

$$
\begin{gathered}
\sigma_{m}=5 \varepsilon^{-1 / 2} \AA^{2}, \\
m_{0}=4 \mathrm{amu}, \quad T_{0}=0 \mathrm{~K}, \\
E / n_{0}=1 \mathrm{Td},
\end{gathered}
$$

where the energy $\varepsilon$ is in $\mathrm{eV}$. Table I shows the eigenvalues from both numerical solution of Eq. (26) and analytical ex- 
TABLE I. Eigenvalue spectra $\left|K_{n}^{\star}\right|\left(10^{-2}\right)$ for the Maxwell model (32). The first and second rows for a given magnetic field strength are from numerical solution of Eq. (26) and analytical expression (30), respectively.

\begin{tabular}{ccccccc}
\hline \hline & \multicolumn{6}{c}{$n$} \\
$\begin{array}{c}B / n_{0} \\
(\mathrm{Hx})\end{array}$ & 0 & 1 & 2 & 3 & 4 & 5 \\
\cline { 2 - 7 } 0 & 0 & 2.968 & 3.363 & 3.494 & 3.563 & 3.608 \\
& 0 & 2.968 & 3.366 & 3.491 & 3.545 & 3.574 \\
169 & 0 & 5.937 & 6.731 & 6.982 & 7.113 & 7.189 \\
& 0 & 5.937 & 6.732 & 6.982 & 7.091 & 7.149 \\
500 & 0 & 29.08 & 32.98 & 34.23 & 34.89 & 35.27 \\
& 0 & 29.07 & 32.97 & 34.19 & 34.72 & 35.00 \\
\hline \hline
\end{tabular}

pression (30) for varying magnetic field strength. We see excellent agreement between the theory and numerical data. Note that in the presence of a magnetic field, the magnitude of $K_{n}^{\star}$ is amplified by a factor of $\left[1+\left(\Omega / \nu_{m}\right)^{2}\right]$ which is 2 and $\sim 10$ for $B / n_{0}=169 \mathrm{Hx}$ and $500 \mathrm{Hx}$, respectively. The larger $K_{n}^{\star}$ implies that spatial relaxation proceeds faster than the electric-field-only case. Physically, under the influences of a magnetic field, a charged particle experiences more elastic collisions in traveling a given linear distance due to gyrations induced by the magnetic field. Therefore, energy transfer between charged particles and neutral gases (i.e., the loss of energy from the swarm particles to the neutral gas) is enhanced and a reduction in relaxation length follows directly.

We expect this general behavior of shorter relaxation length in the presence of an additional magnetic field to carry over to cases when other types of collisions such as inelastic collisions are present.

\section{B. Electrons in a model gas: Inelastic step function cross section}

To understand the fundamental effects of the magnetic field on relaxation characteristics when inelastic collisions are present, we study here electrons in a model gas, using an inelastic step function model. This model was employed previously when studying eigenvalue spectra in the case of electric field only, and the model details are [6]

$$
\begin{gathered}
\sigma_{m}=6 \AA^{2}, \\
\sigma_{i}=0.1 \AA^{2}, \quad \varepsilon_{i}=2 \mathrm{eV}, \\
m_{0}=4 \mathrm{amu}, \quad T_{0}=0 \mathrm{~K},
\end{gathered}
$$

where the inelastic collisions are characterized by a cross section $\sigma_{i}$ and a threshold $\varepsilon_{i}$. Here the electric field strength of interest varies between $0.5 \mathrm{Td}$ and $20 \mathrm{Td}$. When the magnetic field is absent, a "window" of electric field strength was observed [6] for which transport properties exhibit oscillatory behavior as they relax to the spatially uniform state. We study here the effects of varying the magnetic field strength on $K_{1}^{\star}$ and the associated electric field "window" and relaxation characteristics.
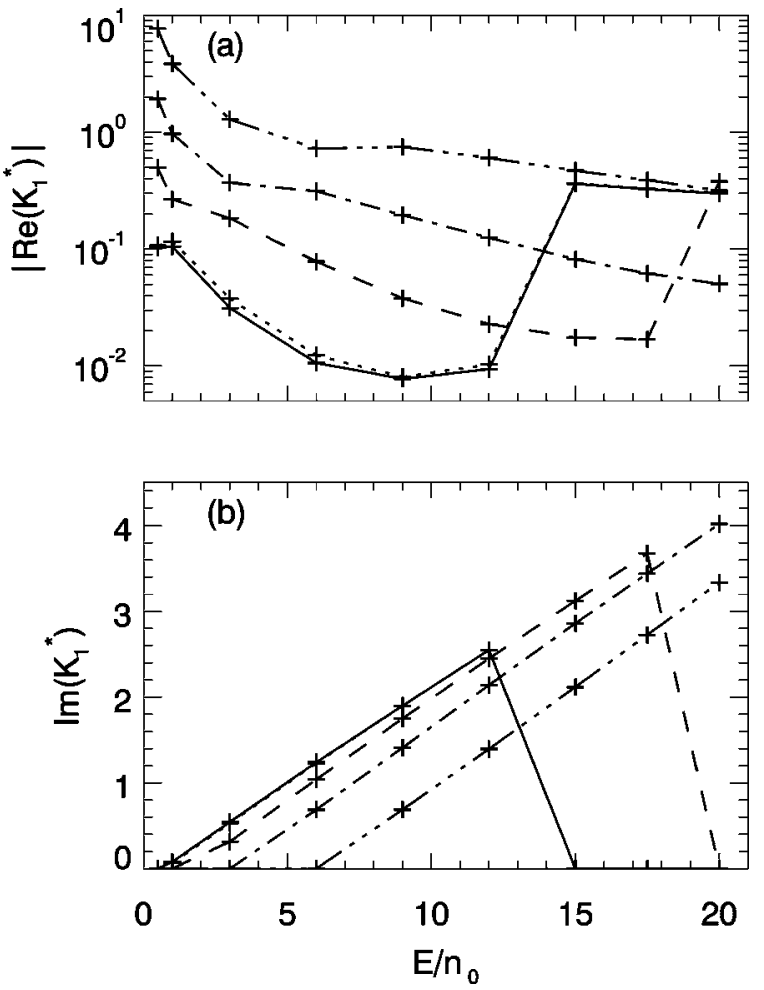

FIG. 2. The real part (a) and imaginary part (b) of $K_{1}^{\star}$ for the inelastic step-function cross-section model (33) over a range of electric field strength for varying magnetic field. Solid line: $B / n_{0}$ $=0$. Dotted line: $B / n_{0}=100 \mathrm{Hx}$. Dashed line: $B / n_{0}=500 \mathrm{Hx}$. Dotdashed line: $B / n_{0}=1000 \mathrm{Hx}$. Dot-dot-dashed line: $B / n_{0}=2000 \mathrm{Hx}$.

Figure 2 shows the variation of $K_{1}^{\star}$ in the above $E / n_{0}$ range while the magnetic field strength varies. We see from Figs. 2(a) and 2(b) that the presence of a magnetic field shifts both $\left|\operatorname{Re}\left(K_{1}^{\star}\right)\right|$ and $\operatorname{Im}\left(K_{1}^{\star}\right)$ toward larger $E / n_{0}$; meanwhile, $\left|\operatorname{Re}\left(K_{1}^{\star}\right)\right|$ is moved upward while $\operatorname{Im}\left(K_{1}^{\star}\right)$ downward.

In the case of electric field only, Fig. 2(b) shows the presence of a window of electric field strength with lower and upper boundaries about $0.5 \mathrm{Td}$ and $12.5 \mathrm{Td}$, respectively, over which nonvanishing $\operatorname{Im}\left(K_{1}^{\star}\right)$ exists. Inside this window, $\left|\operatorname{Re}\left(K_{1}^{\star}\right)\right|$ in Fig. 2(a) is minimal, consistent with our previous work [6]. For cases when a magnetic field is present and inelastic collisions dominate the energy loss processes, as discussed in Sec. III A, a charged particle experiences more elastic collisions in passing a given linear distance. Therefore, energy transfer between the charged particles and the neutral gas is enhanced. To accumulate sufficient energy to reach the inelastic threshold, charged particles thus need to travel a longer linear distance than in the magnetic-field-free case. It follows that the period of spatial oscillations increases when a magnetic field is present, as indicated by the smaller $\operatorname{Im}\left(K_{1}^{\star}\right)$ in Fig. 2(b). The larger $\left|\operatorname{Re}\left(K_{1}^{\star}\right)\right|$ in Fig. 2(a) in the presence of the magnetic field and its increase with $B / n_{0}$ implies that the discussions in Sec. III A in association with Table I carry over to the present situation where both elastic and inelastic collisions are present, so the characteristic relaxation length decreases. For instance, Fig. 2 shows that at $E / n_{0}=6 \mathrm{Td}, P=5.1 \lambda$ and $\Lambda=94 \lambda$ for $B / n_{0}=0$, but when $B / n_{0}=1000 \mathrm{Hx}, P=9.1 \lambda$ and $\Lambda=3.2 \lambda$. Such observa- 

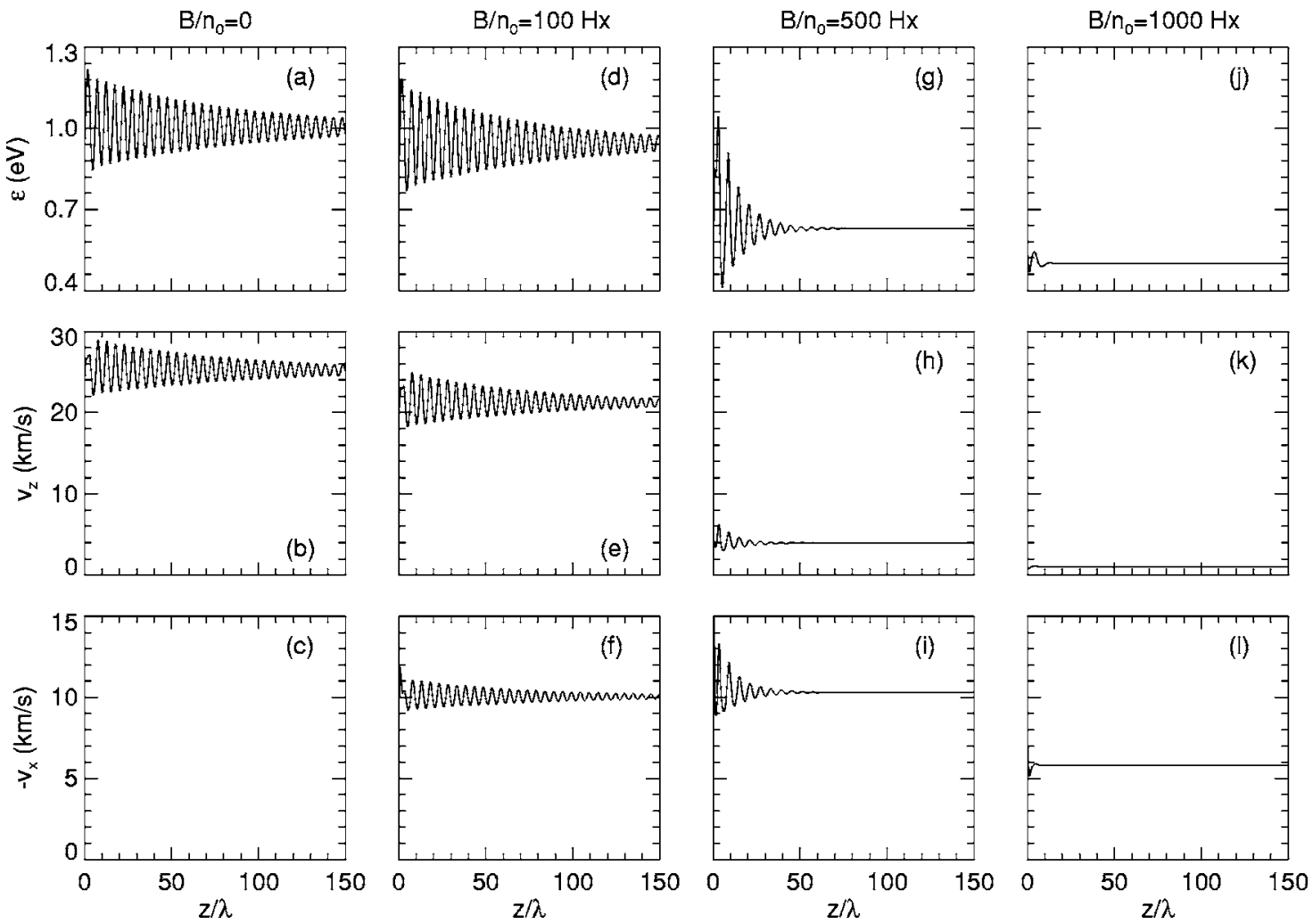

FIG. 3. Spatial relaxation of the mean energy $\varepsilon$ (first row) and average velocities $v_{z}$ (second row) and $v_{x}$ (third row) for the step function collision model (33) at $E / n_{0}=6 \mathrm{Td}$ for a range of magnetic fields: $B / n_{0}=0$ (first column), $100 \mathrm{Hx}$ (second column), $500 \mathrm{Hx}$ (third column), and $1000 \mathrm{Hx}$ (fourth column). The disturbing source is introduced via (18) with $T_{i}=4.0 \times 10^{3} \mathrm{~K}$ and $v_{i}=2.0 \times 10^{4} \mathrm{~m} / \mathrm{s}$.

tions are consistent with an early work for electrons in neon [21].

The shifts of the window toward larger $E / n_{0}$ in both $\left|\operatorname{Re}\left(K_{1}^{\star}\right)\right|$ and $\operatorname{Im}\left(K_{1}^{\star}\right)$ with the application of a magnetic field in Fig. 2 is due to the magnetic cooling effects [32]. First, we consider the shift of the lower boundary of the window. For relaxation which is originally periodic when only an electric field present, the application of a magnetic field of sufficiently large strength reduces the mean energy of charged particles to far below the inelastic threshold. Spatial evolution of the particles is thus dominated by elastic collisions and relaxation is monotonic. Consequently, an $E / n_{0}$ value originally inside the window for periodic relaxation when $B / n_{0}=0$ is now driven outside the window. For example, Fig. 2(b) shows that the lower boundary of $\sim 0.5 \mathrm{Td}$ of the electric field window for $B / n_{0}=0$ is outside the window for $B / n_{0}=500 \mathrm{Hx}$. Second, we consider cases when the relaxation characteristic is monotonic in the presence of only a strong electric field with strength greater than the upper boundary of the window. The reality of $K_{1}$ for strong electric fields is due to the fact that the mean energy is much greater than the inelastic collision threshold, and hence the losses of energy due to inelastic collisions can be treated as a continuous process [46-48]. When an additional magnetic field is introduced, for sufficiently strong magnetic field, the mean energy is now comparable with the inelastic threshold. Energy losses via inelastic collisions dominate, and so oscillatory relaxation proceeds. For instance, Fig. 2(b) shows that the upper boundary of the electric field window is $\sim 12 \mathrm{Td}$ in the absence of a magnetic field. When $B / n_{0}=500 \mathrm{Hx}$, the upper boundary of the window is $\sim 17.5 \mathrm{Td}$ and a shift toward larger $E / n_{0}$ is evident.

To characterize the effects of the magnetic field on relaxation processes in neon, in Ref. [21], the relaxation length and period were parametrized via an empirical formula for damped periodic evolutions with spatial position. This is equivalent to approximately subtracting the information for the eigenvalue $K_{1}$ from the complex electron relaxation profiles, rather than evaluating $K_{1}$ rigorously as in this work.

Figure 3 shows the spatial relaxation of transport properties at $E / n_{0}=6 \mathrm{Td}$ for varying $B / n_{0}$, for a disturbing source introduced via Eq. (18) with $T_{i}=4.0 \times 10^{3} \mathrm{~K}$ and $v_{i}=2.0$ $\times 10^{4} \mathrm{~m} / \mathrm{s}$. In the presence of an electric field only, Figs. 3(a)-3(c) show that both the mean energy and the average velocity in the electric field direction relax toward a spatially uniform state via oscillatory decay, as expected from the study of $K_{1}$ in Fig. 2. The relaxation of these quantities is very slow, taking approximately $500 \lambda$ to reach to the spatially uniform state. When $B / n_{0}=100 \mathrm{Hx}$, we see from Figs. $3(\mathrm{~d})-3(\mathrm{f})$ that both the maximal and spatially uniform values of $\varepsilon$ and $v_{z}$ are lower than the case when $B=0$, due to magnetic cooling effects and gyrations of the electrons caused by the magnetic field [32]. In addition, by virtue of the crossed magnetic field drift the velocity $v_{x}$ is now nonzero and in the $-x$ direction, due to electron gyrations [32]. Moreover, Figs. 3(d)-3(f) show that the period of oscillations is almost the same as in the $B=0$ case and so is the relaxation length. These are consistent with the results in Fig. 2. Figures $3(\mathrm{~g})-3(\mathrm{i})$ show that when the magnetic field strength is in- 

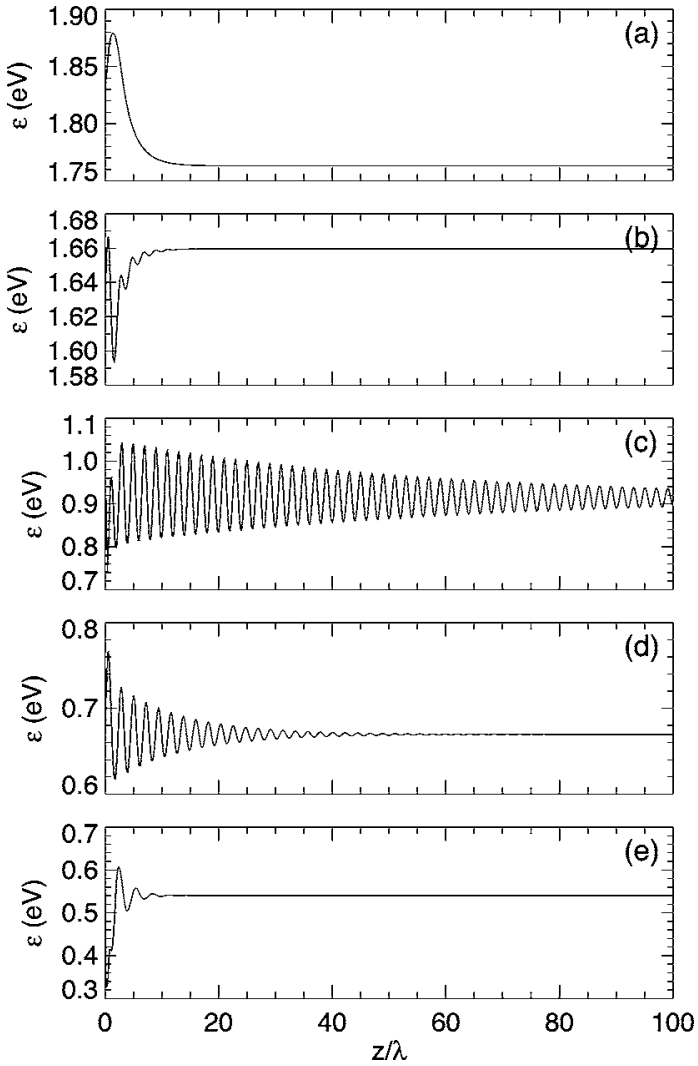

FIG. 4. Spatial relaxation of the mean energy for the step function collision model (33) at $E / n_{0}=15 \mathrm{Td}$ for a range of magnetic fields: (a) $B / n_{0}=0$, (b) $B / n_{0}=100 \mathrm{Hx}$, (c) $B / n_{0}=500 \mathrm{Hx}$, (d) $B / n_{0}=1000 \mathrm{Hx}$, and (e) $B / n_{0}=2000 \mathrm{Hx}$. The disturbing source is introduced via (18) with $T_{i}=8.0 \times 10^{3} \mathrm{~K}$ and $v_{i}=2.0 \times 10^{3} \mathrm{~m} / \mathrm{s}$.

creased to $500 \mathrm{Hx}$, significant changes in all the quantities occur. First, both $\varepsilon$ and $v_{z}$ are much lower than for the case of $100 \mathrm{Hx}$, while $v_{x}$ is enhanced in both the maximal and spatially uniform values. Second, the period of oscillation is larger and agrees with the Fig. 2 prediction. The relaxation proceeds much quicker and taking approximately $70 \lambda$ to be fully relaxed. Figures 3(j)-3(1) show the case when the magnetic field is further increased up to $1000 \mathrm{Hx}$. We see that the mean energy is now much less than the threshold energy of the inelastic collision process, energy losses via inelastic collisions are reduced, and elastic collisions are enhanced. The relaxation profiles actually show very weak irregular oscillations, which are quickly damped. Under such conditions, all the quantities are significantly lower than the magnetic-fieldfree case.

To study the shifts of the window of electric field strengths when a magnetic field is present, as predicted by eigenvalue analysis, Fig. 4 shows the relaxation of the mean energy at $E / n_{0}=15 \mathrm{Td}$ for various $B / n_{0}$. The disturbing source has $T_{i}=8.0 \times 10^{3} \mathrm{~K}$ and $v_{i}=2.0 \times 10^{3} \mathrm{~m} / \mathrm{s}$. Figure 4(a) shows that in the absence of a magnetic field the relaxation profile is aperiodic and relaxation proceeds very fast (fully relaxed at $z / \lambda \approx 15$ ). The aperiodic relaxation occurs because losses of energy via inelastic collisions can be approximated as continuous processes as discussed in relation to Fig. 2. Figure 4(b) shows that the application of a mag- netic field of strength $100 \mathrm{Hx}$ causes little change in the relaxation length although the mean energy is slightly less than in Fig. 4(a). However, Fig. 4(b) also shows that the mean energy varies irregularly before it has relaxed. When this occurs the disturbing source introduced at $z=0$ essentially causes all the eigenmodes to contribute to the relaxation profiles for regions near the disturbance. Although $K_{1}^{\star}$ is real, some of the other eigenvalues $K_{n}^{\star}(n \neq 0,1)$ are complex and the amplitudes of their real parts may be comparable with $\operatorname{Re}\left(K_{1}^{\star}\right)$. Consequently, weak oscillations contributed by these eigenmodes are superimposed on the regular relaxation controlled mainly by the $K_{1}^{\star}$ eigenmode. Figure 4(c) shows that when $B / n_{0}=500 \mathrm{Hx}$, significant changes occur. The relaxation is dramatically slower than for the lower $B / n_{0}$, and it takes over $300 \lambda$ to reach the spatially uniform state. Moreover, Fig. 4(c) shows that oscillatory relaxation prevails. The period of oscillations is $P \approx 2.0 \lambda$, larger than the value $(1.89 \lambda)$ predicted via the classical relation $\varepsilon_{i} / e E$ if energy loss in elastic collisions is negligible. Due to the magnetic cooling effects, the mean energy of the electrons reduces. For this particular $B / n_{0}$, the mean energy is reduced to a level that energy losses by inelastic collisions become discrete again. In addition, the probability for the occurrence of inelastic collisions is significant; hence, energy loss by inelastic collisions dominates that by elastic collisions. The transport properties thus evolve periodically to the spatially uniform states. Furthermore, we find that both the relaxation length and period of oscillations are consistent with the eigenvalue analysis predicted by Fig. 2 . Figure 4(d) shows that when the magnetic field strength is further increased to 1000 $\mathrm{Hx}$, the mean energy relaxes faster than for $500 \mathrm{Hx}$ but still slower than for the other two weaker magnetic field strengths, although relaxation is still characterized by periodic oscillations. For $B / n_{0}$ greater than $500 \mathrm{Hx}$, energy losses by elastic collisions become important, so relaxation is faster and the oscillation period is slightly larger than in Fig. 4(c). Figure 4(e) shows, for $B / n_{0}=2000 \mathrm{Hx}$, that periodic relaxations are damped out to the spatially uniform state much more quickly than for $1000 \mathrm{Hx}$. For even stronger magnetic fields, the mean energy is much lower (not shown) than the inelastic threshold. Loss of energy through inelastic collisions is insignificant, and elastic collisions dominate energy losses. Relaxation is aperiodic, and once again the electric field strength is pushed outside the window.

The main results of this section can be summarized as follows. In general, the presence of a magnetic field can both speed up and slow down spatial relaxation of transport properties. When the original relaxation characteristics in the presence of electric field only, be they monotonic or periodic, are unchanged by the application of a magnetic field, relaxation proceeds faster and the period of oscillations is larger if relaxation is oscillatory. For the cases when the original relaxation characteristics are changed by the magnetic field, relaxation occurs slower (faster) if the original relaxation is monotonic (periodic). Moreover, at sufficiently high magnetic field strengths, relaxation is always monotonic and faster than when only the electric field exists. The magnetic field can thus shift the window of electric field strength and affect greatly the corresponding electron relaxation characteristics. 




FIG. 5. Anisotropic elastic and isotropic inelastic cross sections of methane [14]. Dotted line: $\sigma_{0}-\sigma_{1}$. Dashed line: $\sigma_{0}-\sigma_{2}$. Dotdashed line: $\sigma_{0}-\sigma_{3}$. Dot-dot-dashed line: $\sigma_{v 1, v 3}$. Long-dashed line: $\sigma_{v 2, v 4}$.

\section{Electrons in methane}

In this section, we consider electron spatial relaxation in methane gas, for which the elastic cross section is characterized by a Ramsauer minimum. The onset of inelastic collisions occurs at energies near this minimum, and thus the inelastic cross section is not small compared with the elastic cross sections in this energy region. This was the example studied in the original multiterm theory developed by Lin $e t$ al. [29] and is thus of special interest in the present nonhydrodynamic studies.

The cross sections used here are those of Schmidt [14] and are shown in Fig. 5. In these cross sections, anisotropic elastic collision cross sections are employed with the first three partial cross section differences $\sigma_{0}-\sigma_{l}(l \leqslant 3)$ specified. For $l>4$, we assume $\sigma_{0}-\sigma_{l}=\sigma_{0}-\sigma_{3}$. The inelastic collisions given in Ref. [14] assume isotropic scattering. We also neglect ionization, attachment, and rotational excitations, which is appropriate for the field strengths under consideration.

We first consider a very low electric field $E / n_{0}=0.5 \mathrm{Td}$; the methane gas has a temperature $T_{0}=293 \mathrm{~K}$ as in our previous work [7], while varying the magnetic field strength. For this choice $E / n_{0}$ in the absence of a magnetic field, our early work showed that the relaxation is periodic and undergoes the maximum spatial distance before it settles down to the spatially uniform state [7]. Figure 6(a) shows that the spatial evolution of mean energy is periodic and weakly damped; it takes about $800 \lambda$ to reach the uniform state. The period of oscillations agrees with prediction via the classical relation $\varepsilon_{i} / e E$, where $\varepsilon_{i}=0.162 \mathrm{eV}$ is the threshold energy of the first vibrational excitation. For this $E / n_{0}$, energy losses through elastic collisions and other inelastic channels are minor by comparison. We see from Figs. 6(b) and 6(c) that the presence of a magnetic field acts to heavily damp the oscillations and relaxation proceeds much faster than the magnetic-field-free case in Fig. 6(a). This occurs because of the decrease of the mean energy as $B / n_{0}$ rises, enhances the energy losses due to elastic collisions, and they become comparable with those due to inelastic collisions. Hence the re-
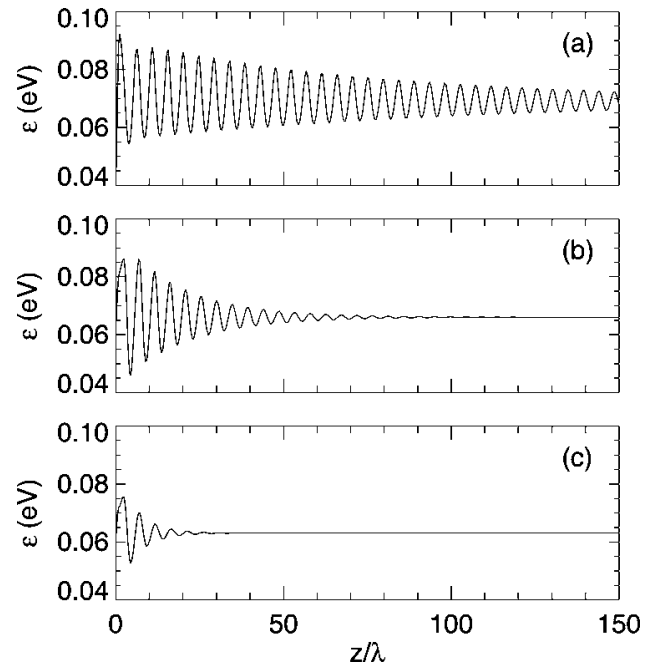

FIG. 6. Spatial relaxation of the mean energy in methane at $E / n_{0}=0.5 \mathrm{Td}$ for a range of magnetic fields: (a) $B / n_{0}=0$, (b) 100 $\mathrm{Hx}$, and (c) $200 \mathrm{Hx}$. The disturbing source is introduced via (18) with $T_{i}=2.0 \times 10^{2} \mathrm{~K}$ and $v_{i}=2.0 \times 10^{3} \mathrm{~m} / \mathrm{s}$.

laxation profile is periodic but quickly damped. In addition, Figs. 6(a)-6(c) show that the period of oscillations becomes longer as $B / n_{0}$ increases. We find that both the relaxation length and period are consistent with eigenvalue analysis.

Now we extend our investigation to a higher electric field strength $E / n_{0}=5 \mathrm{Td}$ and consider $B / n_{0}=0,30 \mathrm{Hx}$, and 200 Hx. The methane gas temperature now is $295 \mathrm{~K}$. This condition is the same as in previous work on hydrodynamic electron transport in methane $[33,34]$. In these studies, it was found that the mean energy is in the vicinity of the Ramasauer minimum in the elastic cross sections. The velocity distribution in this energy region is significantly anisotropic, and the two-term approximation fails. Motivated by these previous findings, the aim here is to test the present approaches for nonhydrodynamic spatial relaxation. The disturbing source is represented by the drifted Maxwellian (18) with $T_{i}=5 \times 10^{3} \mathrm{~K}$ and $v_{i}=10^{4} \mathrm{~m} / \mathrm{s}$.

Figure 7 shows the relaxation of mean energy and average velocities under such conditions. In the presence of an electric field only, Figs. 7(a)-7(c) show that a two-term approximation results in large error in all three quantities: $\sim 7 \%$ in $\varepsilon$ near the source region $z / \lambda<1, \sim 7 \%$ in $v_{z}$ accross the whole spatial regions, and serious error in $v_{x}$ for $z / \lambda<2$. Actually, a four-term calculation is required to achieve an accuracy of $2 \%$ or better, although a three-term approximation is sufficient for hydrodynamic conditions [33].

With the application of a weak magnetic field at $30 \mathrm{Hx}$, Figs. 7(d)-7(f) show that the two-term approximation yields results in large error in both $\varepsilon$ and $v_{z}$ (e.g., $15 \%$ in $v_{z}$ ) and significant error in $v_{x}$ when near the disturbing source. In this case, a four-term approximation is still needed to accurately account for the relaxation profile near the origin, even though a three-term truncation is adequate for regions where hydrodynamic conditions prevail, in agreement with an early study [33]. When the magnetic field is increased to $200 \mathrm{Hx}$, Figs. 7(g)-7(i) show that a two-term approximation is adequate for the system under hydrodynamic conditions. This 

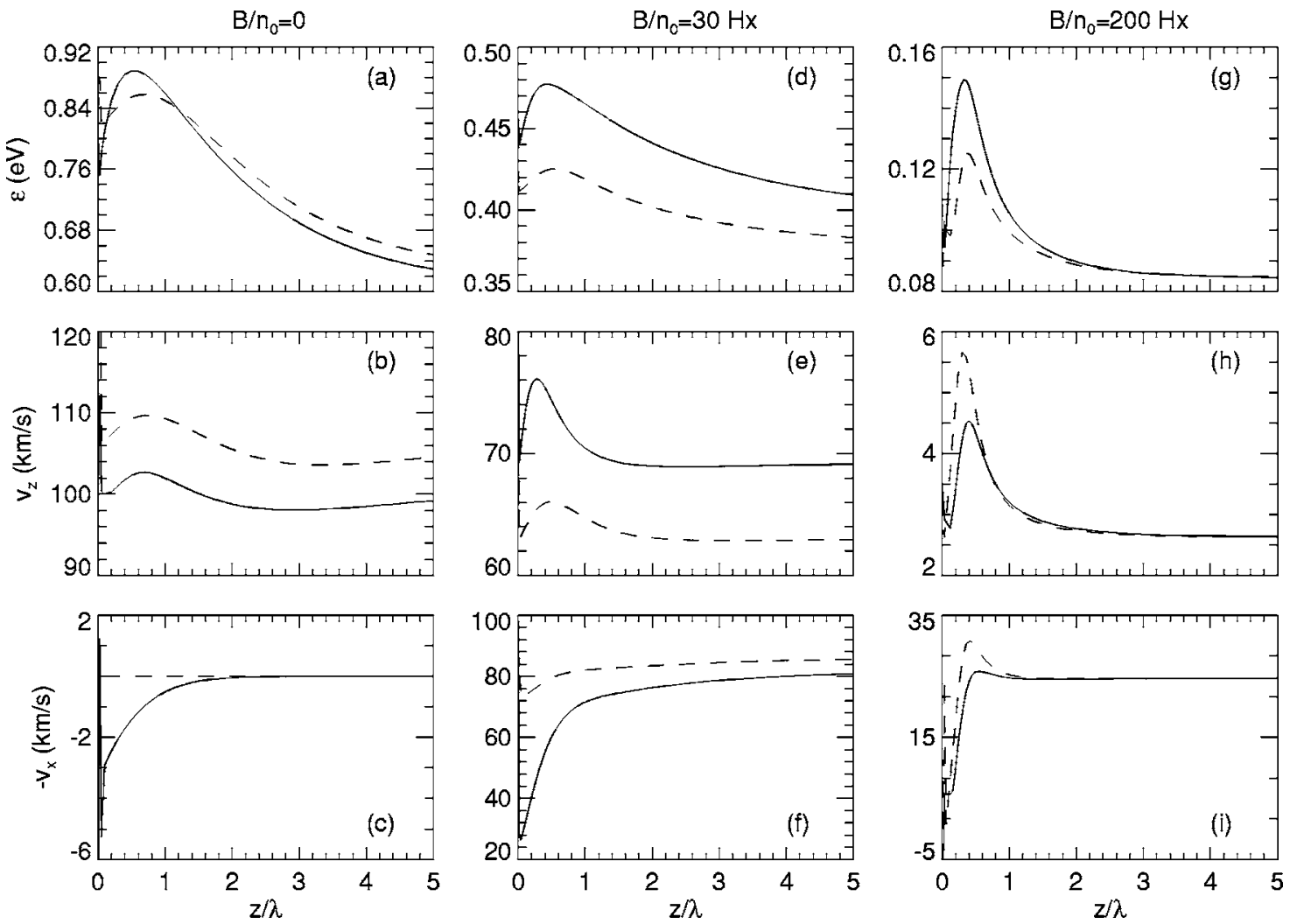

FIG. 7. Spatial relaxation of the mean energy $\varepsilon$ (first row), average velocities $v_{z}$ (second row), and $v_{x}$ (third row) in methane gas at $E / n_{0}=5 \mathrm{Td}, B / n_{0}=0$ (first column), $30 \mathrm{Hx}$ (second column), and $200 \mathrm{Hx}$ (third column), respectively. The disturbing source is introduced via (18) with $T_{i}=3 \times 10^{3} \mathrm{~K}$ and $v_{i}=10^{4} \mathrm{~m} / \mathrm{s}$. The dashed curves are for a two-term approximation; the solid curves represent converged results with a four-term approximation.

observation is consistent with a previous work [34] which showed a reduction of anisotropy in the electron distribution function with increasing $B / n_{0}$ and, hence, a lower-order truncation with $l_{\max }=1$ becoming sufficient. However, Figs. 7(g)-7(i) also show that near the source region, a four-term approximation is essential in furnishing converged mean energy and average velocities within desired accuracy. The requirement of multiterm analysis for regions near the source and at the low magnetic field strengths observed here is consistent with discharge modelings for a real gas [27,28].

\section{CONCLUSION}

We have developed two complementary methods to study the spatial relaxation of charged-particle swarms in an idealized steady-state Townsend experiment, under the influence of uniform orthogonal electric and magnetic fields. In both cases, a "two-temperature" moment technique in which operators in velocity space of the Boltzmann equation represented in terms of Burnett functions has been employed. The numerical methods developed here are suitable for both ions and electrons, but we have presented results for electrons only.

The spatial relaxation characteristics of transport properties were studied both by full numerical solution and by eigenvalue spectral analysis of the Boltzmann equation, respectively. The numerical approaches were first validated against analytical predictions, and we have demonstrated excellent agreement in eigenvalue spectra for the Maxwell elastic collision model. We have also established that monotonic spatial relaxation is shortened when a magnetic field is applied.

When both elastic and inelastic collisional processes are present, the application of a magnetic field was found to significantly alter the relaxation characteristics. Generally, the magnetic field can both enhance or retard the spatial relaxation of transport properties. When the original qualitative relaxation characteristics, be they monotonic or periodic for the magnetic-field-free case, are unchanged by the application of a magnetic field, relaxation proceeds quicker, and the period of oscillations is longer, if relaxation is periodic. When the original relaxation characteristics are, on the other hand, altered qualitatively by the presence of a magnetic field, relaxation proceeds slower (faster) if the original relaxation is monotonic (periodic). Furthermore, for sufficiently strong magnetic field strengths, relaxation is always monotonic and proceeds faster than when only the electric field is present. In summary, the magnetic field can shift the window of the electric field strength in which oscillatory evolution occurs and modify the corresponding relaxation characteristics, both qualitatively and quantitatively.

The importance of multiterm analysis was illustrated by considering electrons in methane. We found that, generally, a multiterm calculation is required to correctly account for the spatial profiles of transport properties near the disturbing 
source, even though a two-term approximation may be sufficient in the hydrodynamic regime in the presence of a strong magnetic field.

Finally, we found that the equivalent electric field concept as used in the spatially homogeneous case is inappropriate under nonhydrodynamic conditions. Moreover, although this is a theoretical work, we would like to encourage experimentalists to look for the effects described by developing a "Franck-Hertz experiment with a magnetic field." In our opinion, the photon flux technique of Fletcher [49] may offer the best and least intrusive means of making such observations.

\section{ACKNOWLEDGMENT}

The research was supported by Denison Small Grant, School of Physics, University of Sydney.

\section{APPENDIX: EIGENVALUE SPECTRUM ANALYSIS FOR THE MAXWELL ELASTIC COLLISION MODEL}

For the case of electron swarms undergoing only elastic collisions which are governed by an isotropic differential cross section, the collision operator has the form [50,51]

$$
\begin{gathered}
J^{0}=-\frac{m}{m_{0} c^{2}} \frac{d}{d c}\left[c^{2} \nu_{m}\left(c+\frac{k T_{0}}{m} \frac{d}{d c}\right)\right], \\
J^{l}=\nu_{l}=\nu_{m} \quad(l \geqslant 1) .
\end{gathered}
$$

If the eigenfunction of Eq. (26) is represented by an expansion in spherical harmonics similar to Eq. (2), i.e.,

$$
\psi(\boldsymbol{c})=\sum_{l=0}^{\infty} \sum_{m=-l}^{l} \psi_{m}^{(l)}(z, c) Y_{m}^{[l]}(\hat{\boldsymbol{c}}),
$$

and the two-term approximation is made, Eq. (26) becomes, using the $J^{l}$ above,

$$
\begin{gathered}
J^{0} \psi_{0}^{(0)}+i \frac{a}{\sqrt{3} c^{2}} \frac{\partial}{\partial c}\left[c^{2} \psi_{0}^{(1)}\right]+i \frac{c}{\sqrt{3}} K \psi_{0}^{(1)}=0, \\
J^{1} \psi_{-1}^{(1)}+\frac{\Omega}{\sqrt{2}} \psi_{0}^{(1)}=0, \\
J^{1} \psi_{0}^{(1)}-i \frac{a}{\sqrt{3}} \frac{\partial}{\partial c} \psi_{0}^{(0)}+\frac{\Omega}{\sqrt{2}}\left[\psi_{1}^{(1)}-\psi_{-1}^{(1)}\right]-i \frac{c}{\sqrt{3}} K \psi_{0}^{(0)}=0, \\
J^{1} \psi_{1}^{(1)}-\frac{\Omega}{\sqrt{2}} \psi_{0}^{(1)}=0 .
\end{gathered}
$$

Eliminating $\psi_{-1}^{(1)}$ and $\psi_{1}^{(1)}$ between Eqs. (A4)-(A7) and assuming a cold gas $\left(T_{0}=0\right)$, we have

$$
-\frac{m}{m_{0} c^{2}} \frac{\partial}{\partial c}\left[c^{3} \nu_{m} \psi_{0}^{(0)}\right]+i \frac{a}{\sqrt{3} c^{2}} \frac{\partial}{\partial c}\left[c^{2} \psi_{0}^{(1)}\right]+i \frac{c}{\sqrt{3}} K \psi_{0}^{(1)}=0,
$$

$$
\nu_{m}\left[1+\left(\frac{\Omega}{\nu_{m}}\right)^{2}\right] \psi_{0}^{(1)}-i \frac{a}{\sqrt{3}} \frac{\partial}{\partial c} \psi_{0}^{(0)}-i \frac{c}{\sqrt{3}} K \psi_{0}^{(0)}=0 .
$$

To represent Eqs. (A8) and (A9) in terms of real quantities, we perform a transformation

$$
\Psi_{l m}=i^{l}\left[\frac{(2 l+1)(l-|m|) !}{4 \pi(l+|m|) !}\right]^{1 / 2} \psi_{m}^{(l)}
$$

so that the eigenvalue problems above become

$$
-\frac{m}{m_{0} c^{2}} \frac{\partial}{\partial c}\left[c^{3} \nu_{m} \Psi_{00}\right]+\frac{a}{3 c^{2}} \frac{\partial}{\partial c}\left[c^{2} \Psi_{10}\right]+\frac{c}{3} K \Psi_{10}=0,
$$

$$
\nu_{m}\left[1+\left(\frac{\Omega}{\nu_{m}}\right)^{2}\right] \Psi_{10}+a \frac{\partial}{\partial c} \Psi_{00}+c K \Psi_{00}=0 .
$$

Following the procedures employed by Robson [52] when studying boundary effects on a distribution function, for an arbitrary function $G(c)$, we introduce the transformation

$$
\|G(c)\|_{s}=\int_{0}^{\infty} \exp \left[-\frac{1}{2} s c^{2}\right] G(c) d c,
$$

where $s$ may be complex. Note that the following identity holds:

$$
\left\|c^{2} G(c)\right\|_{s}=-2 \frac{\partial}{\partial s}\|G(c)\|_{s} .
$$

In terms of the Laplace transform operator $L_{s}$ defined below

$$
\|G(c)\|_{s}=\int_{0}^{\infty} \exp (-s x) \frac{G(\sqrt{2 x})}{\sqrt{2 x}} d x \equiv L_{s}\left\{\frac{G(\sqrt{2 x})}{\sqrt{2 x}}\right\},
$$

where $x=c^{2} / 2$, Eq. (A14) can be written as

$$
\left\|c^{2} G(c)\right\|_{s} \equiv L_{s}\{\sqrt{2 x} G(\sqrt{2 x})\} .
$$

Multiplying Eqs. (A11) and (A12) by $c^{j} \exp \left(-s c^{2} / 2\right)$ and $c^{j^{\prime}} \exp \left(-s c^{2} / 2\right)$, respectively, and integrating over $c$ yields

$$
\begin{gathered}
\frac{1}{3} a(2-j)\left\|c^{j-1} \Psi_{10}\right\|_{s}+\frac{1}{3}(a s+K)\left\|c^{j+1} \Psi_{10}\right\|_{s} \\
=\frac{m}{m_{0}}\left\{s\left\|\nu_{m} c^{j+2} \Psi_{00}\right\|_{s}+(2-j)\left\|\nu_{m} c^{j} \Psi_{00}\right\|_{s}\right\}, \\
j^{\prime} a\left\|c^{j^{\prime}-1} \Psi_{00}\right\|_{s}-(a s+K)\left\|c^{j^{\prime}+1} \Psi_{00}\right\|_{s} \\
=\left\|\nu_{m}\left[1+\left(\Omega / \nu_{m}\right)^{2}\right] c^{j^{\prime}} \Psi_{10}\right\|_{s},
\end{gathered}
$$

where $j$ and $j^{\prime}$ are arbitrary integers. For the Maxwell model, we choose $j=2$ and $j^{\prime}=3$, and also define $\kappa$ via

$$
K=-\kappa a .
$$

Eliminating terms with $\Psi_{10}$ between Eqs. (A17) and (A18) and using relation (A14) we find, after some algebra, 


$$
\left\|c^{2} \Psi_{00}\right\|_{s, \kappa}=\eta \frac{\left(s+\xi^{-}\right)^{3(p-1) / 2}}{\left(s+\xi^{+}\right)^{3 p / 2}},
$$

where the subscript $\kappa$ has been added to denote the eigenfunction corresponding to the eigenvalue $K, \eta$ is a constant of integration, and

$$
\begin{gathered}
\xi^{ \pm} \equiv \frac{1}{2} \beta^{2}\left[1-2 \kappa / \beta^{2} \pm\left(1-4 \kappa / \beta^{2}\right)^{1 / 2}\right], \\
p \equiv \frac{1+\left(1-4 \kappa / \beta^{2}\right)^{1 / 2}}{2\left(1-4 \kappa / \beta^{2}\right)^{1 / 2}}, \\
\beta^{2} \equiv \frac{3 m}{m_{0}}\left[1+\left(\frac{\Omega}{\nu_{m}}\right)^{2}\right]\left(\frac{\nu_{m}}{a}\right)^{2} .
\end{gathered}
$$

Note that $\left\|c^{2} \Psi_{00}\right\|_{s, \kappa}$ is the Laplace transform of $\sqrt{2 x} \Psi_{00}(\sqrt{2 x})$, so

$$
\sqrt{2 x} \Psi_{00}(\sqrt{2 x})=L_{s}^{-1}\left\{\left\|c^{2} \Psi_{00}\right\|_{s, \kappa}\right\} .
$$

In the case of $\kappa=0$, Eq. (A20) reduces to

$$
\left\|c^{2} \Psi_{00}\right\|_{s, \kappa=0}=\eta\left(s+\beta^{2}\right)^{-3 / 2} .
$$

Using the definition (A13) and relation (A14), it follows that

$$
\Psi_{00}(c, \kappa=0)=\sqrt{\frac{2}{\pi}} \eta \exp \left[-\frac{1}{2} \beta^{2} c^{2}\right] .
$$

As the eigenmode with $\kappa=0$ corresponds to the spatially uniform state, the eigenfunction $\Psi_{00}(c, \kappa=0)$ thus represents the spatially homogeneous distribution, which is a Maxwellian at temperature $T \equiv m / k \beta^{2}$.

The downstream behavior is determined by those eigenvalues for which $\kappa \geqslant 0(K \leqslant 0)$; hence, it follows from Eqs. (A21) and (A22) that

$$
p \geqslant 1, \quad \xi^{+}>0, \quad \xi \geqslant 0 .
$$

In order for the inverse Laplace transform (A24) to be regular at the origin $c=0$, we must have

$$
\frac{3}{2}(p-1)=n \quad(n=0,1,2, \ldots),
$$

i.e.,

$$
p=1+\frac{2}{3} n,
$$

leading to a quantization of the eigenvalues. Thus, substituting Eq. (A29) into Eq. (A22) yields the allowed value of $\kappa$,

$$
\kappa_{n}=\frac{2 n(2 n+3)}{(4 n+3)^{2}} \beta^{2},
$$

and the eigenvalues are given by

$$
K_{n}=-\frac{6 n(2 n+3)}{(4 n+3)^{2}} \frac{m}{m_{0}} \frac{\nu_{m}^{2}}{a}\left[1+\left(\frac{\Omega}{\nu_{m}}\right)^{2}\right] .
$$

Note that the above analytical expression for $K_{n}$ can be used as a benchmark for numerical calculations.
[1] R. Winkler, S. Arndt, D. Loffhagen, F. Sigeneger, and D. Uhrlandt, Contrib. Plasma Phys. 44, 437 (2004).

[2] R. Winkler, G. Petrov, F. Sigeneger, and D. Uhrlandt, Plasma Sources Sci. Technol. 6, 118 (1997).

[3] G. Petrov and R. Winkler, J. Phys. D 30, 53 (1997).

[4] F. Sigeneger and R. Winkler, Plasma Chem. Plasma Process. 17, 1 (1997).

[5] F. Sigeneger and R. Winkler, Plasma Chem. Plasma Process. 17, 281 (1997).

[6] R. E. Robson, B. Li, and R. D. White, J. Phys. B 33, 507 (2000).

[7] B. Li, R. D. White, and R. E. Robson, J. Phys. D 35, 2914 (2002)

[8] E. A. Mason and E. W. McDaniel, Transport Properties of Ions in Gases (Wiley, New York, 1988).

[9] L. A. Viehland and E. A. Mason, Ann. Phys. (N.Y.) 91, 499 (1975); 110, 287 (1978).

[10] M. A. Liberman and A. J. Lichtenberg, Principles of Plasma Discharge and Material Processing (Wiley Interscience, Hoboken, NJ, 2005).

[11] W. Blum and L. Rolandi, Particle Detection with Drift Chambers (Springer, Berlin, 1993).

[12] Z. Ceplecha, J. Borovicka, W. G. Elford, D. O. Revelle, R. L. Hawkes, V. Porubcan, and M. Simek, Space Sci. Rev. 84, 327 (1998).
[13] J. Li and Q. M. Chen, J. Phys. D 26, 1541 (1993).

[14] B. Schmidt, J. Phys. B 24, 379 (1991).

[15] K. Kumar, H. R. Skullerd, and R. E. Robson, Aust. J. Phys. 33, 343 (1980).

[16] E. Maride and J. P. Boeuf (unpublished).

[17] R. E. Robson, R. D. White, and Z. Lj. Petrovic, Rev. Mod. Phys. 77, 1303 (2005).

[18] R. E. Robson, Aust. J. Phys. 28, 523 (1975).

[19] K. Kumar, J. Phys. D 14, 2199 (1981).

[20] S. B. Vehovac and Z. Lj. Petrovic, Aust. J. Phys. 52, 999 (1999).

[21] R. Winkler, V. A. Maiorov, and F. Sigeneger, J. Appl. Phys. 87, 2708 (2000).

[22] D. Uhrlandt and R. Winkler, IEEE Trans. Plasma Sci. 29, 462 (2001).

[23] I. A. Porokhova, Yu. B. Golubovskii, J. Bretagne, M. Tichy, and J. F. Behnke, Phys. Rev. E 63, 056408 (2001).

[24] I. A. Porokhova, Yu. B. Golubovskii, and J. F. Behnke, Phys. Rev. E 71, 066406 (2005).

[25] R. E. Robson, R. Winkler, and F. Sigeneger, Phys. Rev. E 65, 056410 (2002).

[26] K. F. Ness, Phys. Rev. E 47, 327 (1993).

[27] I. A. Porokhova, Yu. B. Golubovskii, and J. F. Behnke, Phys. Rev. E 71, 066407 (2005).

[28] I. A. Porokhova, M. Holik, O. Bilyk, P. Kudrna, Yu. B. Gol- 
ubovskii, M. Tichy, and J. F. Behnke, Contrib. Plasma Phys. 45, 319 (2005).

[29] S. L. Lin, R. E. Robson, and E. A. Mason, J. Chem. Phys. 71, 3483 (1979).

[30] R. Winkler, Adv. At., Mol., Opt. Phys. 43, 19 (2000).

[31] R. E. Robson and K. F. Ness, Phys. Rev. A 33, 2068 (1986).

[32] K. F. Ness, J. Phys. D 27, 1848 (1994).

[33] K. F. Ness, Aust. J. Phys. 48, 557 (1999).

[34] R. D. White, K. F. Ness and R. E. Robson, J. Phys. D 32, 1842 (1999).

[35] R. Winkler, D. Loffhagen, and F. Sigeneger, Appl. Surf. Phys. 192, 50 (2002).

[36] C. S. Wang-Chang, G. E. Uhlenbeck, and J. DeBoer, Studies in Statistical Mechanics, Vol. II (Wiley, New York, 1964).

[37] K. F. Ness and R. E. Robson, Phys. Rev. A 34, 2185 (1986).

[38] S. Chapman and T. G. Cowling, The Mathematical Theory of Non-uniform Gases (Cambridge University Press, Cambridge, England, 1939).

[39] R. D. White, R. E. Robson, and K. F. Ness, Comput. Phys. Commun. 142, 349 (2001).

[40] L. Tonks, Phys. Rev. 51, 744 (1937).

[41] A. E. D. Heylen, Electrical Ionization and Breakdown of Gases in a Crossed Magnetic Field, IEE Proceedings A: Physical Science, Measurement and Instrumentation, Management, and Education, Reviews (IEE, United Kingdom, 1980), Vol. 127, p. 221.

[42] R. E. Robson, Aust. J. Phys. 47, 279 (1994).

[43] R. E. Robson, M. Hildebrandt, and B. Schmidt, Nucl. Instrum. Methods Phys. Res. A 394, 779 (1997).

[44] B. Li, R. D. White, and R. E. Robson, Ann. Phys. (N.Y.) 292, 179 (2001).

[45] N. Shimura and T. Makabe, Appl. Phys. Lett. 62, 678 (1993); 65, 31 (1994).

[46] K. G. Muller, Z. Phys. 169, 432 (1962).

[47] L. D. Landau and E. M. Lifshitz, Quantum Mechanics-Nonrelativistic Theory (Addison-Wesley, Reading, MA, 1965).

[48] Y. M. Li, in Nonequlibruim Effects in Ions and Electron Transport, edited by J. W. Gallagher, D. F. Hudson, E. E. Kunhardt and R. J. V. Brunt (Plenum, New York, 1990), p. 99.

[49] J. Fletcher, J. Phys. D 18, 221 (1985).

[50] W. P. Allis, in Handbuch Der Physik, edited by S. Flugge (Springer-Verlag, Berlin 1956), Vol. 21, p. 383.

[51] E. H. Holt and R. E. Haskell, Foundations of Plasma Dynamics (MacMillan, New York, 1965).

[52] R. E. Robson, Aust. J. Phys. 34, 223 (1981).

[53] R. E. Robson, K. F. Ness, G. E. Sneddon, and L. A. Viehland, J. Comput. Phys. 92, 213 (1991). 